\title{
Successful pregnancy after transient ovarian failure following treatment of symptomatic leiomyomata
}

\author{
Peter Kovacs, M.D., ${ }^{a}$ John J. Stangel, M.D., ${ }^{\mathrm{b}}$ Nanette F. Santoro, M.D., ${ }^{\mathrm{a}}$ and \\ Harry Lieman, M.D. ${ }^{\text {a }}$ \\ Albert Einstein College of Medicine, Division of Reproductive Endocrinology and Infertility, Bronx, New York
}

Received August 20, 2001; revised and accepted November 19, 2001.

Reprint requests: Peter Kovacs, M.D., Division of Reproductive

Endocrinology, Department of Obstetrics and Gynecology, Albert Einstein College of Medicine, 1300 Morris Park Avenue, Mazer Building, Room 313, Bronx, New York 10461 (FAX: 718-430-8586; E-mail: pkovcs@aol.com).

a Division of Reproductive Endocrinology and Infertility, Department of Obstetrics and Gynecology, Albert Einstein College of Medicine.

${ }^{b}$ Westchester Affiliate of Institute for Reproductive Medicine and Science of Saint Barnabas, Livingston, New Jersey.

0015-0282/02/\$22.00 PII S0015-0282(02)03091-1

Objective: To report a case of transient ovarian failure after treatment of symptomatic leiomyomata and review other iatrogenic causes of transient ovarian failure.

Design: Case report and literature review.

Setting: University-affiliated private practice.

Patient(s): A 35-year-old woman with symptomatic leiomyomata.

Intervention(s): Bilateral uterine artery embolization with subsequent abdominal myomectomy to treat unchanged regular heavy menstrual flow.

Main Outcome Measure(s): Ovarian function.

Result(s): Because medical therapy failed to control her menorrhagia, the patient proceeded with uterine artery embolization. She had persistent menorrhagia after bilateral uterine artery embolization and underwent exploratory laparotomy and myomectomy. After surgery, she had amenorrhea, hot flushes, and elevated FSH levels for 3 months. Ovarian function recovered after a short course of oral contraceptives, and the patient conceived without assistance.

Conclusion(s): Several interventions can affect normal ovarian function and can lead to permanent or transient ovarian failure. Possible causes of transient ovarian failure are radioactive iodine treatment, radiation, chemotherapy, pelvic surgery, stress, and uterine artery embolization. Before these interventions are applied, the possibility of ovarian failure and available preventive measures should be discussed with the patient. (Fertil Steril ${ }^{\circledR}$ 2002;77:1292-5. (02002 by American Society for Reproductive Medicine.)

Key Words: Transient ovarian failure, uterine artery embolization, myomectomy, FSH

The ovaries serve a dual purpose during the reproductive years: They produce steroid hormones and release oocytes, both in a cyclical fashion. In most women, menopause occurs around the age of 51 years; however, in unusual cases, the ovaries fail earlier. This failure is generally considered "premature" if it happens before 40 years of age.

There are several known causes of premature ovarian failure, but most cases are idiopathic. When the ovaries fail during the early reproductive years, the condition can often be reversed, as shown by elevated estradiol levels, return of menses, or, in rare cases, successful pregnancy (1).

We report on a 35-year-old woman who was surgically treated for symptomatic leiomyo- mata and developed transient ovarian failure after surgery, but later successfully conceived.

\section{CASE REPORT}

A 35-year-old woman, gravida 1 para 0 , underwent treatment for symptomatic leiomyomata. She presented to us in December 1999 with a long history of chronic pelvic pain and menorrhagia. Her menstrual periods lasted for 7-10 days, with heavy flow.

The patient had previously received medical treatment for endometriosis (oral contraceptive pills and danazol) followed by surgery (laparoscopic ablation). Neither treatment was successful in controlling her symptoms. She had also had an ectopic gestation that was treated with laparoscopic salpingectomy. She was known to have a leiomyomatous uterus. 
On our initial assessment in December 1999, hysterosonography showed a $20 \times 27 \times 19 \mathrm{~mm}$ submucous myoma and several similar-sized intramural myomas and a $27 \times 30 \times 33 \mathrm{~mm}$ simple left ovarian cyst. Her hemoglobin level was $10.1 \mathrm{~g} / \mathrm{dl}$; prothrombin time, partial thromboplastin time, thyroid function tests, and prolactin were within normal limits, and her serum $\beta$-hCG was negative.

Medical therapy, surgery, and uterine artery embolization (UAE) for the myomas were discussed with the patient. She desired future fertility and wanted to conceive in the near future. Since medical treatment had not improved her symptoms in the past and she wanted to avoid another surgical procedure, the patient chose UAE. She was informed about the unknown effects of UAE on subsequent fertility.

Angiography before UAE demonstrated a blood supply to the myomas from the right ovarian artery. The presence of this blood supply can render the UAE unsuccessful; however, this vessel was not embolized because the patient desired fertility.

In March 2000, the patient underwent an uneventful bilateral UAE. Polyvinyl alcohol particles $500 \mu \mathrm{m}$ in size were used. The patient stayed overnight for pain management.

The patient started having regular periods 4 weeks after UAE; however, her heavy menstrual flow remained unchanged. Repeated hysterosonography did not show shrinkage of myomas. At this point, surgical removal of the myomas was recommended and in July 2000, the patient underwent an exploratory laparotomy, lysis of adhesions, and multiple myomectomy.

The surgery was performed under general anesthesia. Tourniquet or clamps on the ovarian vessels were not used. A dilute solution of vasopressin was used to reduce blood loss. Two 3-cm subserosal myomas were removed from the anterior surface, one from the midline and one from the right superior fundal region. A $3-\mathrm{cm}$ submucous myoma was removed through the same anterior incision. During removal of this myoma, the endometrial cavity was entered. Two posterior subserosal myomas measuring $3 \mathrm{~cm}$ and a $3-\mathrm{cm}$ intramural myoma were also removed through a vertical posterior uterine incision. The myometrial defects were closed by using several layers of delayed absorbable suture.

Adhesiolysis around the left adnexa was performed by using electrocautery; the right fallopian tube and ovary were free of adhesions. Continuous irrigation was used during the procedure, and hemostasis was good at completion. The anterior uterine surface was covered with Interceed at the end of the surgery. No surgical complications occurred.

Six weeks after surgery, the patient returned because of amenorrhea and hot flushes. A serum $\beta$-hCG test was negative. The serum FSH level was 87.7 U/L. Observation was attempted for 1 month to see whether the patient's menses would return, but the hot flushes, amenorrhea, and elevated FSH level (22.3 U/L) persisted.
The next month, the patient reported heavy vaginal bleeding lasting more than 1 week and reduced frequency and intensity of hot flushes. A serum $\beta$-hCG test was negative, and the patient was given oral contraceptives for cycle control. Her FSH level was 9.5 U/L.

After 1 month of contraceptive treatment, the patient had normal withdrawal bleeding but had no return of menses for 6 weeks. At this time, her $\beta$-hCG level was positive, and transvaginal ultrasonography showed an early intrauterine pregnancy. She is currently in the second trimester of an uneventful pregnancy.

\section{DISCUSSION}

From the 20th gestational week, the germ-cell pool decreases, and almost complete exhaustion of the pool occurs by menopause, mainly owing to atresia. During the reproductive years, cyclic stimulation of a cohort of follicles, of which only one will ovulate and all others will undergo atresia, contributes to the process of follicle exhaustion. Once no more follicles can be recruited, despite stimulation from the pituitary, a woman reaches menopause (2).

Certain toxic effects and genetic conditions (e.g., radiation, some chemotherapeutic agents, and Turner syndrome) can hasten the depletion of the germ cell pool. Other possible etiologies are immune disease, enzyme defects (e.g., galactosemia), viral infection, smoking, and inadequate gonadotropin secretion (3). Approximately $1 \%$ of women reach menopause prematurely for no known reason (4). Under unusual circumstances, an ovary that was once thought to be failing may regain its activity and produce sufficient steroid hormones and oocytes.

Raymond et al. reported that radioactive iodine-131 ablation therapy for thyroid cancer resulted in amenorrhea with hot flushes within the first year in 18 of 66 women (5). All of the women had elevated FSH levels and decreased estradiol levels. Amenorrhea resolved spontaneously in all cases within 6 to 12 months. One successful pregnancy was reported during follow-up (5).

Women undergoing bone marrow transplantation for malignant hematologic diseases receive total body irradiation and chemotherapy before the procedure. Such therapy destroys both developing and resting follicles. Several studies report a high rate of amenorrhea with menopausal symptoms immediately after treatment (6-8). Sanders et al. reported that return of ovarian function was more likely after chemotherapy alone compared with chemotherapy plus radiation therapy (7). Schimmer et al. found that recovery of ovarian function was more likely among younger patients (8).

Certain chemotherapeutic agents alone are toxic to the ovaries. Alkylating agents (cyclophosphamide, chlorambucil, busulfan, nitrogen mustard, vinblastine, and mitomycin-D) have been linked to ovarian dysfunction $(9,10)$. Of 
these agents, cyclophosphamide has been studied extensively. Several studies have reported permanent ovarian failure after use of cyclophosphamide, but various rates of recovery of ovarian function have also been reported $(11,12)$. These differences are most likely due to different doses, duration of treatment, and patient age. Successful pregnancies have been reported among women who had return of ovarian function.

Transient loss of ovarian function has also been described in cases of major emotional stress (13). Netter et al. reported hypergonadotropic hypogonadism secondary to emotional stress (e.g., death in the family or divorce) in 10 women 18 to 38 years of age. Only one of these patients had subsequent return of menses (13).

Ovarian failure after UAE has been described. Uterine artery embolization has been shown to be effective in the management of symptomatic leiomyomata $(14,15)$. Failure of the procedure has been attributed to coexisting adenomyosis (16) and collateral blood supply from ovarian or other pelvic vessels $(17,18)$. Uterine arteries anastomose with the ovarian arteries at the hilum of the ovary. This vascular connection may be responsible for nontargeted embolization and compromise of the ovarian blood supply.

Permanent ovarian failure has been described after bilateral UAE (19); however, most of these patients were older than 45 years of age. Nonetheless, a few cases have been reported of transient loss of ovarian function, as evidenced by amenorrhea, menopausal symptoms, and elevated FSH level, followed by recurrence of cyclic menstrual bleeding and disappearance of menopausal symptoms $(14,20)$. It is unknown whether polyvinyl alcohol particles used for embolization have direct toxic effects on the ovary.

The effect of pelvic-adnexal surgery on ovarian function has also been evaluated $(21,22)$. Ranney and Chastain found no difference in reproductive function after adnexal surgery in 223 patients (21). Sayegh and Garcia reported on one case of transient ovarian failure after partial ovarian resection and two cases of permanent failure after adnexal surgery. However, the incidence of menstrual irregularities was similar in women after surgery compared with women who had not had surgery (22). Bowman et al. analyzed the effect of pelvic adhesions on ovarian function by assessing baseline FSH levels. They showed a positive correlation between the extent of adhesions and baseline FSH levels but found no difference between women with and without adhesions (23). Boyers et al. reported that among 19 patients with premature ovarian failure, 7 had had surgery 6 months to 8 years before the onset of amenorrhea, suggesting a possible role of surgery itself (24). Although no prospective studies of ovarian function and gonadotropin levels before and after pelvicadnexal surgery have been done, some evidence indicates that such surgery sometimes affects ovarian function.

Our patient underwent UAE and abdominal myomectomy to treat symptomatic uterine myomata. She reported regular menstrual cycles with unchanged persistent menorrhagia starting 4 weeks after UAE. In her case, UAE probably failed because of collateral vascular supply of the uterus from the ovarian arteries, as was predicted by angiography before the procedure. Unilateral ovarian artery embolization was not done at the time of UAE owing to her desire for future fertility. However, the patient reported amenorrhea and hot flushes only after abdominal surgery. At this time, she had elevated FSH levels on repeated occasions. After 3 months of amenorrhea, hot flushes, and elevated FSH levels, she had an episode of metrorrhagia that was controlled by oral contraceptives. After 1 month of oral contraceptive use, she conceived naturally.

While we cannot rule out idiopathic premature ovarian failure in her case, the sequence of events implies that the two procedures performed back to back influenced her ovarian function. The patient was asymptomatic, with regular menstrual cycles and no hot flushes, before the interventions. However, her FSH levels had not been monitored before UAE. The two procedures together probably compromised the blood flow to the ovary. It takes several months to develop sufficient collateral blood supply. In the 3 months after surgery, the patient's FSH level was elevated but was decreasing, perhaps reflecting improved blood flow to the ovary. Once the "nutritional" input was sufficient for the follicles to advance from the resting phase to the growth phase, the patient showed clinical evidence of recovered ovarian function.

Temporary return of ovarian function, as indicated by elevated estradiol levels, follicle development, and even pregnancy, has been described often in women with idiopathic or iatrogenic ovarian failure $(1,3,4)$. Jones proposed that ovarian function returns when resting follicles that were "protected by their inactivity" resume their cycle and acquire gonadotropin sensitivity (25). To date, no scientific evidence supports this proposal, and there is no widely accepted explanation of return of ovarian function after temporary failure. Recovery after interventions that compromise ovarian blood supply would seem to be possible if sufficient collateral circulation develops.

There are very few interventions that one can offer to patients before a procedure that may affect ovarian function. Studies evaluating the effect of GnRH agonists before such therapy are promising, but data in humans are currently limited $(26,27)$. Lateral transposition of ovaries has been shown to be beneficial if radiation is limited to the paraaortic nodes (28). Other options include cryopreservation of ovarian tissue with subsequent in vitro culturing of oocytes or transplantation of the thawed tissue. If time allows, patients can undergo IVF cycles with cryopreservation of embryos before radiation or chemotherapy $(27,29)$.

About $8 \%$ to $10 \%$ of women with idiopathic premature ovarian failure achieve pregnancy over long follow-up $(3,4)$. 
Many of these women conceive after hormone therapy for menopausal symptoms. To date, no interventions (GnRH agonist suppression, estradiol, ovarian hyperstimulation, or glucocorticoids) seem to improve this outcome, except for IVF with donor oocytes (30-32).

In summary, we present a case of transient ovarian failure after back-to-back UAE and abdominal myomectomy. The possibility of iatrogenic ovarian failure after UAE or pelvic surgery merits careful consideration in patients who desire future fertility.

\section{References}

1. Rebar RW, Erickson GF, Yen SSC. Idiopathic premature ovarian failure: clinical and endocrine characteristics. Fertil Steril 1982;37:3541 .

2. Adashi EY, Rock JA, Rosenwaks Z. Reproductive endocrinology, surgery and technology. Volume 1. Philadelphia: Lippincott-Raven; 1996:18-37.

3. Lieman H, Santoro N. Premature ovarian failure: a modern approach to diagnosis and treatment. Endocrinologist 1997;7:314-21.

4. Anasti JN Premature ovarian failure: an update. Fertil Steril 1998;70: $1-15$.

5. Raymond JP, Izembart M, Marliac V, Dagousset F, Merceron RE, Vulpillat M, et al. Temporary ovarian failure in thyroid cancer patients after thyroid remnant ablation with radioactive iodine. J Clin Endocrinol Metab 1989;69:186-9.

6. Spinelli S, Chiodi S, Bacigalupo A, Brasca A, Valenzano Menada M, Ravera $\mathrm{G}$, et al. Ovarian recovery after total body irradiation and allogenic bone marrow transplantation: long-term follow up of 79 females. Bone Marrow Transplant 1994;14:373-80.

7. Sanders JE, Buckner CD, Amos D, Levy W, Appelbaum FR, Doney K, et al. Ovarian function following marrow transplantation for aplastic anemia or leukemia. J Clin Oncol 1988;6:813-8

8. Schimmer AD, Quatermain M, Imrie K, Ali V, McCrae J, Stewart AK, et al. Ovarian function after autologous bone marrow transplantation. J Clin Oncol 1998;16:2359-63.

9. Reichman BS, Green KB. Breast cancer in young women: effect of chemotherapy on ovarian function, fertility and birth defects. Monogr Natl Cancer Inst 1994;16:125-9.

10. Choo YC, Chan SY, Wong LC, Ho K. Ovarian dysfunction in patients with gestational trophoblastic neoplasia treated with short intensive courses of etoposide (VP-16-213). Cancer 1985;55:2348-52.

11. Kumar R, Biggart JD, McEvoy J, McGeown MG. Cyclophosphamide and reproductive function. Lancet 1972;1:1212-4.

12. Warne GL, Fairley KF, Hobbs JB, Martin FIR. Cyclophosphamideinduced ovarian failure. N Engl J Med 1973;289:1159-62.

13. Netter A, Lambert A, Lumbroso P. Etudes sur les amenorrhees. Les amenorrhees ovarioplegiques. Bull Mem Soc Med Hop Paris 1958;74: $248-50$.
14. Spies JB, Scialli AR, Jha RC, Imaoka I, Ascher SM, Fraga VM, et al. Initial results from uterine fibroid embolization for symptomatic leiomyomata. J Vasc Interv Radiol 1999;10:1149-57.

15. Goodwin SC, McLucas B, Lee M, Chen G, Perrella R, Vedantham S, et al. Uterine artery embolization for the treatment of uterine leiomyomata midterm results. J Vasc Interv Radiol 1999;10:1159-65.

16. Smith SJ, Sewall LE, Handelsman A. A clinical failure of uterine fibroid embolization due to adenomyosis. J Vasc Interv Radiol 1999; 10:1171-4.

17. Pelage JP, Le Dref O, Jacob D, Rymer R. Ovarian artery supply of uterine fibroid. J Vasc Interv Radiol 2000;11:535.

18. Nikolic B, Spies JB, Abbara S, Goodwin SC. Ovarian artery supply of uterine fibroids as a cause of treatment failure after uterine artery embolization: a case report. J Vasc Interv Radiol 1999;10:1167-70.

19. Stringer NH, Grant T, Park J, Oldham L. Ovarian failure after uterine artery embolization for treatment of myomas. J Am Assoc Gynecol Laparosc 2000;7:395-400.

20. Amato P, Roberts AC. Transient ovarian failure: a complication of uterine artery embolization. Fertil Steril 2001;75:438-9.

21. Ranney B, Chastain D. Ovarian function, reproduction and later operations following adnexal surgery. Obstet Gynecol 1978;51:521-7.

22. Sayegh R, Garcia CR. Ovarian function after conservational ovarian surgery: a long-term follow-up study. Int J Gynecol Obstet 1992;39: 303-9.

23. Bowman MC, Cooke ID, Lenton EA. Investigation of impaired ovarian function as a contributing factor to infertility in women with pelvic adhesions. Hum Reprod 1993;8:1654-6.

24. Boyers SP, Luborsky JL, DeCherney AH. Usefulness of serial measurements of serum follicle stimulating hormone, luteinizing hormone and estradiol in patients with premature ovarian failure. Fertil Steril 1988;50:408-12.

25. Jones GS. Corpus luteum: composition and function. Fertil Steril 1990; 54:21-6.

26. Blumenfeld $Z$. Ovarian rescue/protection from chemotherapeutic agents. J Soc Gynecol Investig 2001;8:S60-4.

27. Posada MN, Kolp L, Garcia JE. Fertility options for female cancer patients: facts and fiction. Fertil Steril 2001;75:647-53.

28. Baker JW, Morgan RL, Peckham MJ, Smithers DW. Preservation of ovarian function in patients requiring radiotherapy for para-aortic and pelvic Hodgkin's disease. Lancet 1972;1:1307-8.

29. Van Kasteren Y. Treatment concepts for premature ovarian failure. J Soc Gynecol Investig 2001;8:S58-9.

30. Nelson LM, Kimzey LM, White BJ, Merriam GR. Gonadotropin suppression for the treatment of karyotypically normal spontaneous premature ovarian failure: a controlled trial. Fertil Steril 1992:57:50-5.

31. Taylor AE, Adams JM, Mulder JE, Martin KA, Sluss PM, Crowley WF Jr. A randomized, controlled trial of estradiol replacement therapy in women with hypergonadotropic amenorrhea. J Clin Endocrinol Metab 1996;81:3615-21.

32. Van Kasteren Y, Braat DDM, Hemrika DJ, Lambalk CB, RekersMombarg LTM, Blomberg BME, et al. Corticosteroids do not influence ovarian responsiveness to gonadotropins in patients with premature ovarian failure: a randomized, placebo-controlled trial. Fertil Steril 1999;71:90-5. 